\title{
ANALYSIS OF SOLUTIONS OF TIME FRACTIONAL TELEGRAPH EQUATION
}

\author{
R. JOICE NIRMALA AND K. BALACHANDRAN
}

Department of MAthematics, Bharathiar University, CoImbatore-641046, India.

E-mail address: joicenirmala26@gmail.com, kb.math.bu@gmail.com

\begin{abstract}
In this paper, the solution of time fractional telegraph equation is obtained by using Adomain decomposition method and compared with various other method to determine the efficiency of Adomain decomposition method. These methods are used to obtain the series solutions. Finally, results are analysed by plotting the solutions for various fractional orders.
\end{abstract}

\section{INTRODUCTION}

The main motivation to study time fractional telegraph equation is that, it would be difficult to imagine a world without communication systems. Normally transmission media can be categorized into two groups, namely guided and unguided transmission lines. In order to optimize guided communication systems, it is necessary to determine or project power and signal losses in the system, since all the system has such losses. To determine these losses and eventually ensure a maximum output, it is necessary to formulate some kind of equation with which to calculate these losses. This motivates us to study the analysis of time fractional telegraph equation.

Here, the Adomain decomposition method (ADM) is used to construct the approximate solution of time fractional telegraph equation. The result of this study reveal that the Adomain decomposition method is very powerful, effective, and quiet accurate. It was introduced by Adomian in 1980, which provides an effective procedure for explicit and numerical solutions of a wide class of differential systems representing real physical problems. This method is efficiently utilized for initial or boundary value problems, for linear or nonlinear, ordinary or partial differential equations and even for stochastic systems as well. Moreover no linearisation or perturbation is required in this method. During the last two decades, extensive work has been done using ADM as it provides analytical approximate solutions for nonlinear equations and considerable interest in solving fractional differential equations has been stimulated.

There are many methods, namely Laplace and Fourier transforms, have been utilized to solve linear fractional differential equations (FDE) [23, 24, 28]. In contrast for solving the nonlinear

Received by the editors November 23 2013; Revised April 19 2014; Accepted in revised form April 292014 ; Published online June 92014.

2010 Mathematics Subject Classification. 35R11.

Key words and phrases. Adomain decomposition method(ADM), decomposition method by Laplace transformation, Fractional telegraph equation, Mittag-Leffler function, Caputo derivative. 
FDE, one has to depend upon numerical solutions [3, 12, 33]. Recently developed technique of Adomian decomposition [2] has proven to be a powerful method and has successfully been applied in a variety of problems. Biazar et al. [7] have employed the Adomian decomposition to solve a system of ordinary differential equations and system of Volterra integral equations [6] as well. Wazwaz [31] has explored this method to obtain solutions of wave equation. The classical telegraph equation and space or time fractional telegraph equations have been solved by a number of researchers namely Biazar et al. [6, 8, 7], Cascaval et al. [9], Kaya [19], Momani [22], Odibat and Momani [33], Orsingher and Zhao [25], Orsingher and Beghin [24], Sevimlican [30] and Yildirim [32] using various techniques such as variational iteration method, transform method, Adomian decomposition method, generalized differential transform method, and homotopy perturbation method. Adomian decomposition offers certain advantages over routine numerical methods. Numerical methods use discretization which gives rise to rounding off errors causing loss of accuracy and requires large computer power and time. By using Adomain decomposition method Parthiban and Balachandran [27] analysed the solutions of system of fractional partial differential equations. Daftardar-Gejji and Babakhani [13] have studied the solution of system of FDE and proved the existence and uniqueness theorems for the initial value problem. Following this Daftardar-Gejji and Jafari $[10,11]$ have taken up the problem of finding explicit solutions for system of FDE and have developed a decomposition method for the linear FDE of the form

$$
D^{\alpha_{i}} y_{i}(x)=\sum_{j=1}^{n}\left(\phi_{i j}(x)+\nu_{i j} D^{\alpha_{i j}}\right) y_{j}+g_{i}(x),
$$

with

$$
y_{i}^{(k)}(0)=c_{k}^{i}, 0 \leq k \leq\left[\alpha_{i}\right], 1 \leq i, j \leq n, \alpha_{i}, \alpha_{i j} \in \mathbb{R}^{+} .
$$

It is useful for obtaining closed form or numerical approximation for wide class of stochastic and deterministic problems in science and engineering. This method has been modified by Wazwaz [31].

A mathematical derivation for the time fractional telegraph equation is obtained from classical telegraph equation by replacing the second order time derivative by fractional derivative of order $1<2 \alpha \leq 2$ and by replacing first order time derivative by fractional derivative of order $\frac{1}{2}<\alpha \leq 1$. The modeling of time fractional telegraph equation [8] is as follows.

$$
\begin{array}{r}
\frac{\partial^{2 \alpha} e(x, t)}{\partial t^{2 \alpha}}+(R C+G L) \frac{\partial^{\alpha} e(x, t)}{\partial t^{\alpha}}+\operatorname{GRe}(x, t)=\frac{\partial^{2} e(x, t)}{\partial x^{2}}, \\
\text { where } 0<x<L, t>0, \frac{1}{2}<\alpha \leq 1 .
\end{array}
$$

and

$$
\begin{array}{r}
\frac{\partial^{2 \alpha} i(x, t)}{\partial t^{2 \alpha}}+(R C+G L) \frac{\partial^{\alpha} i(x, t)}{\partial t^{\alpha}}+G R i(x, t)=\frac{\partial^{2} i(x, t)}{\partial x^{2}} \\
\text { where } 0<x<L, t>0, \frac{1}{2}<\alpha \leq 1
\end{array}
$$


where $x$ is the distance from sending end of the cable, $e(x, t)$ is the potential at any point on the cable at any time, $i(x, t)$ is the current at any point on the cable at any time, $R$ is the resistance of the cable, $L$ is the inductance of the cable, $G$ is the conductance to ground, $C$ is the capacitance to ground. In this paper the Adomain decomposition method is used to investigate the time fractional telegraph equation and the analytical solution of the telegraph equation is calculated and compared with other methods. In this the solution is obtained in the form of a series with easily computable components.

\section{PReliminaries}

In this section, we provide some basic definitions of fractional integral and differential operators.

Definition 2.1. The Riemann-Liouville fractional integral operator of order $\alpha>0$ of a function $f \in L^{1}\left(\mathbb{R}^{+}\right)$is defined as

$$
I^{\alpha} f(t)=\frac{1}{\Gamma(\alpha)} \int_{0}^{t}(t-s)^{\alpha-1} f(s) d s
$$

where $\Gamma(\cdot)$ is the Euler gamma function.

Definition 2.2. The Riemann-Liouville fractional derivative of order $\alpha>0, n-1<\alpha<n$, $n \in \mathbb{N}$, is defined as

$$
D^{\alpha} f(t)=D^{n} I^{n-\alpha} f(t)=\frac{1}{\Gamma(n-\alpha)}\left(\frac{d}{d t}\right)^{n} \int_{0}^{t}(t-s)^{n-\alpha-1} f(s) \mathrm{d} s,
$$

where the function $f(t)$ has absolutely continuous derivatives upto order $n-1$.

Definition 2.3. The Caputo fractional derivative of order $\alpha>0, n-1<\alpha<n$, is defined as

$$
{ }^{C} D_{0}^{\alpha} f(t)=\frac{1}{\Gamma(n-\alpha)} \int_{0}^{t}(t-s)^{n-\alpha-1} f^{(n)}(s) \mathrm{d} s
$$

where the function $f(t)$ has absolutely continuous derivative upto order $n-1$.

Definition 2.4. The Caputo fractional derivative of two variables of order $\alpha>0, n-1<$ $\alpha<n$, is defined as

$$
{ }^{C} D_{0}^{\alpha} f(x, t)=\frac{1}{\Gamma(n-\alpha)} \int_{0}^{t}(t-s)^{n-\alpha-1} \frac{\partial f^{n}(x, s)}{\partial s^{n}} \mathrm{~d} s
$$

where the function $f(t)$ has absolutely continuous derivative upto order $n-1$.

Definition 2.5. The Laplace transform of Caputo derivative is defined as

$$
L_{t}\left[D_{t}^{\alpha} u(x, t)\right]=s^{\alpha} u(x, s)-\sum_{k=0}^{n-1} u^{k}(x, 0) s^{\alpha-1-k}, n-1<\alpha \leq n .
$$


The power function of fractional derivative is

$$
\begin{aligned}
I_{t}^{\alpha} D_{t}^{\alpha} f(t) & =f(t)-\sum_{k=0}^{m-1} f^{k}(0) \frac{t^{k}}{k !}, \\
D_{t}^{\alpha} t^{\mu} & =\frac{\Gamma(\mu+1)}{\Gamma(\mu+1-\alpha)} t^{\mu-\alpha}, \mu>-1, \\
I_{t}^{\alpha} t^{\mu} & =\frac{\Gamma(\mu+1)}{\Gamma(\mu+\alpha+1)} t^{\mu+\alpha}, \mu>-1 .
\end{aligned}
$$

The Mittag-Leffler function is defined as follows:

Definition 2.6 (Mittag-Leffler function). The Mittag-Leffler function $E_{\alpha}(z)$ is defined as

$$
E_{\alpha}(z)=\sum_{k=0}^{\infty} \frac{z^{k}}{\Gamma(\alpha k+1)}, z \in \mathbb{C}, \operatorname{Re}(\alpha)>0 .
$$

The Mittag-Leffler function $E_{\alpha, \beta}(z)$ is defined as

$$
E_{\alpha, \beta}(z)=\sum_{k=0}^{\infty} \frac{z^{k}}{\Gamma(\alpha k+\beta)}, z, \beta \in \mathbb{C}, \operatorname{Re}(\alpha)>0 .
$$

Caputo fractional derivative is considered throughout this paper because it provides us the advantage of requiring initial conditions given in terms of integer order derivatives. The time fractional telegraph equation can be solved by various methods. Khan [20] discussed the homotophy pertubation method by Laplace transformation. Huang [16] discussed the solution of time fractional telegraph equation in terms of Laplace and Fourier transform in the variables $\mathrm{t}$ and $\mathrm{x}$, where we get the solution in terms of infinite series not a closed one. In double Laplace transformation method $[5,15]$ we need both initial and boundary conditions to find exact solution, but here we are given only initial conditions. So the remaining method available is Adomain decomposition method and decomposition method by Laplace transformation method.

In the following sections Adomain decomposition and decomposition method by Laplace transformation method is used to obtain homogeneous and nonhomogeneous analytic and approximate solution of time fractional telegraph equation.

\section{Adomain Decomposition Method}

Consider the differential equation of the form

$$
L u+R u+N u=g,
$$

where $L$ is the highest linear order derivative operator and invertible, $R$ is the linear differential operator of order less that $L, N u$ is the nonlinear term and $g$ is the source term

$$
L u=g-R u-N u,
$$


because $L$ is invertible, the equivalent expression is

$$
L^{-1} L u=L^{-1} g-L^{-1} R u-L^{-1} N u \text {. }
$$

If $L$ is a second-order operator, for example, $L^{-1}$ is a two fold integration operator and $L^{-1} L u=u-u(0)-t u^{\prime}(0)$, then yields

$$
u=u(0)+t u^{\prime}(0)+L^{-1} g-L^{-1} R u-L^{-1} N u .
$$

Now the solution $u$ can be presented as a series $u=\sum_{n=0}^{\infty} u_{n}$, with

$$
u_{0}=u(0)+t u^{\prime}(0)+L^{-1} g
$$

and $u_{n}, n>0$ is to be determined. The nonlinear term $N u$ will be decomposed by the infinite series of Adomain polynomials

$$
N u=\sum_{n=0}^{\infty} A_{n}
$$

where $A_{n}$ is calculated using the formula

$$
A_{n}=\frac{1}{n !}\left[\frac{d^{n}}{d \lambda^{n}} N(v(y))\right]_{\lambda=0}, n=0,1,2, \cdots,
$$

where

$$
v(y)=\sum_{n=0}^{\infty} \lambda^{n} u_{n}
$$

Now the series solution $u=\sum_{n=0}^{\infty} u_{n}$ to the differential equation is calculated iteratively as follows:

$$
\begin{aligned}
u_{0} & =u(0)+t u^{\prime}(0)+L^{-1} g, \\
u_{n+1} & =-L^{-1}\left(R u_{n}\right)-L^{-1}\left(A_{n}\right), n \geq 0 .
\end{aligned}
$$

The results reveal that the Adomain decomposition method is very effective and convenient.

3.1. Homogeneous Fractional Telegraph Equation. Let us consider the time fractional telegraph equation $[8,13,16,19]$

$$
\frac{\partial^{\beta} u(x, t)}{\partial t^{\beta}}+a \frac{\partial^{\alpha} u(x, t)}{\partial t^{\alpha}}+b u(x, t)=c^{2} \frac{\partial^{2} u(x, t)}{\partial x^{2}}, t>0,1<\beta \leq 2, \frac{1}{2}<\alpha \leq 1,
$$

with initial conditions

$$
u(x, 0)=f(x), u_{t}(x, 0)=g(x)
$$


By using Adomain decomposition method, we have

$$
\begin{aligned}
& I^{\beta}\left[\frac{\partial^{\beta} u(x, t)}{\partial t^{\beta}}+a \frac{\partial^{\alpha} u(x, t)}{\partial t^{\alpha}}+b u(x, t)\right]=I^{\beta}\left[c^{2} \frac{\partial^{2} u(x, t)}{\partial x^{2}}\right], \\
& I^{\beta}\left[\frac{\partial^{\beta} u(x, t)}{\partial t^{\beta}}\right]=I^{\beta}\left[c^{2} \frac{\partial^{2} u(x, t)}{\partial x^{2}}-a \frac{\partial^{\alpha} u(x, t)}{\partial t^{\alpha}}-b u(x, t)\right],
\end{aligned}
$$

by using (2.5), (2.6) and (2.7) we have

$$
u(x, t)=u(x, 0)+t u_{t}(x, 0)+I^{\beta}\left[c^{2} \frac{\partial^{2} u(x, t)}{\partial x^{2}}-a \frac{\partial^{\alpha} u(x, t)}{\partial t^{\alpha}}-b u(x, t)\right] .
$$

By using the initial conditions, we get

$$
u(x, t)=f(x)+\operatorname{tg}(x)+I^{\beta}\left[c^{2} \frac{\partial^{2} u(x, t)}{\partial x^{2}}-a \frac{\partial^{\alpha} u(x, t)}{\partial t^{\alpha}}-b u(x, t)\right] .
$$

Take

$$
\begin{aligned}
u_{0} & =f(x)+g(x) t \\
u_{n+1} & =I^{\beta}\left[c^{2} \frac{\partial^{2} u_{n}(x, t)}{\partial x^{2}}-a \frac{\partial^{\alpha} u_{n}(x, t)}{\partial t^{\alpha}}-b u_{n}(x, t)\right]
\end{aligned}
$$

Now, by iterating approach, we get

$$
\begin{aligned}
u_{1}(x, t)= & I^{\beta}\left[c^{2} \frac{\partial^{2} u_{0}(x, t)}{\partial x^{2}}-a \frac{\partial^{\alpha} u_{0}(x, t)}{\partial t^{\alpha}}-b u_{0}(x, t)\right] \\
= & I^{\beta}\left[c^{2} f^{(2)}(x)+c^{2} g^{(2)}(x) t-a f(x) \frac{t^{-\alpha}}{\Gamma(1-\alpha)}\right. \\
& \left.-a g(x) \frac{t^{1-\alpha}}{\Gamma(2-\alpha)}-b f(x)-b g(x) t\right]
\end{aligned}
$$

using the definitions (2.5), (2.6) and (2.7), we get

$$
\begin{aligned}
u_{1}(x, t)= & \frac{t^{\beta}}{\Gamma(\beta+1)}\left[c^{2} f^{(2)}(x)-b f(x)\right]+\frac{t^{\beta+1}}{\Gamma(\beta+2)}\left[c^{2} g^{(2)}(x)-b g(x)\right] \\
& -a f(x) \frac{t^{\beta-\alpha}}{\Gamma(\beta-\alpha+1)}-a g(x) \frac{t^{\beta-\alpha+1}}{\Gamma(\beta-\alpha+2)}
\end{aligned}
$$


Next,

$$
\begin{aligned}
u_{2}(x, t)= & I^{\beta}\left[c^{2} \frac{\partial^{2} u_{1}(x, t)}{\partial x^{2}}-a \frac{\partial^{\alpha} u_{1}(x, t)}{\partial t^{\alpha}}-b u_{1}(x, t)\right], \\
= & I^{\beta}\left[\frac{t^{\beta}}{\Gamma(\beta+1)}\left[c^{4} f^{(4)}(x)-2 b c^{2} f^{(2)}+b^{2} f(x)\right]\right. \\
& +\frac{t^{\beta+1}}{\Gamma(\beta+2)}\left[c^{4} g^{(4)}(x)-2 b c^{2} g^{(2)}+b^{2} g(x)\right] \\
& +\frac{t^{\beta-\alpha}}{\Gamma(\beta-\alpha+1)}\left[-2 a c^{2} f^{(2)}(x)+2 a b f(x)\right] \\
& +\frac{t^{\beta-\alpha+1}}{\Gamma(\beta-\alpha+2)}\left[-2 a c^{2} g^{(2)}(x)+2 a b g(x)\right] \\
& \left.+a^{2} f(x) \frac{t^{\beta-2 \alpha}}{\Gamma(\beta-2 \alpha+1)}+a^{2} g(x) \frac{t^{\beta-2 \alpha+1}}{\Gamma(\beta-2 \alpha+2)}\right]
\end{aligned}
$$

Again by definitions (2.5), (2.6) and (2.7) we get

$$
\begin{aligned}
u_{2}(x, t)= & \frac{t^{2 \beta}}{\Gamma(2 \beta+1)}\left[c^{4} f^{(4)(x)}-2 b c^{2} f^{(2)}(x)+b^{2} f(x)\right] \\
& +\frac{t^{2 \beta+1}}{\Gamma(2 \beta+2)}\left[c^{4} g^{(4)(x)}-2 b c^{2} g^{(2)}(x)+b^{2} g(x)\right] \\
& +\frac{t^{2 \beta-\alpha}}{2 \beta-\alpha+1}\left[-2 a c^{2} f^{(2)}(x)+2 a b f(x)\right] \\
& +\frac{t^{2 \beta-\alpha+1}}{2 \beta-\alpha+2}\left[-2 a c^{2} g^{(2)}(x)+2 a b g(x)\right] \\
& +a^{2} f(x) \frac{t^{2 \beta-2 \alpha}}{\Gamma(2 \beta-2 \alpha+1)}+a^{2} g(x) \frac{t^{2 \beta-2 \alpha+1}}{\Gamma(2 \beta-2 \alpha+2)}
\end{aligned}
$$

by proceeding in this manner the solution of the time fractional telegraph equation is of the form

$$
u(x, t)=\sum_{n=0}^{\infty} u_{n}(x, t)=u_{0}(x, t)+u_{1}(x, t)+\cdots
$$


Therefore,

$$
\begin{aligned}
u(x, t)= & \frac{t^{\beta}}{\Gamma(\beta+1)}\left[c^{2} f^{(2)}(x)-b f(x)\right]+\frac{t^{\beta+1}}{\Gamma(\beta+2)}\left[c^{2} g^{(2)}(x)-b g(x)\right] \\
& -a f(x) \frac{t^{\beta-\alpha}}{\Gamma(\beta-\alpha+1)}-a g(x) \frac{t^{\beta-\alpha+1}}{\Gamma(\beta-\alpha+2)} \\
& +\frac{t^{2 \beta}}{\Gamma(2 \beta+1)}\left[c^{4} f^{(4)(x)}-2 b c^{2} f^{(2)}(x)+b^{2} f(x)\right] \\
& +\frac{t^{2 \beta+1}}{\Gamma(2 \beta+2)}\left[c^{4} g^{(4)(x)}-2 b c^{2} g^{(2)}(x)+b^{2} g(x)\right] \\
& +\frac{t^{2 \beta-\alpha}}{2 \beta-\alpha+1}\left[-2 a c^{2} f^{(2)}(x)+2 a b f(x)\right] \\
& +\frac{t^{2 \beta-\alpha+1}}{2 \beta-\alpha+2}\left[-2 a c^{2} g^{(2)}(x)+2 a b g(x)\right] \\
& +a^{2} f(x) \frac{t^{2 \beta-2 \alpha}}{\Gamma(2 \beta-2 \alpha+1)}+a^{2} g(x) \frac{t^{2 \beta-2 \alpha+1}}{\Gamma(2 \beta-2 \alpha+2)}+\cdots .
\end{aligned}
$$

3.2. Nonhomogeneous Fractional Telegraph Equation. Consider the time fractional telegraph equation $[29,32]$ of the form

$$
\frac{\partial^{\alpha} u(x, t)}{\partial t^{\alpha}}+a \frac{\partial^{\alpha-1} u(x, t)}{\partial t^{\alpha-1}}+b u(x, t)=c^{2} \frac{\partial^{2} u(x, t)}{\partial x^{2}}+h(x, t), t>0,1<\alpha \leq 2,
$$

with initial conditions (3.3) by Adomain decomposition method we have

$$
\begin{aligned}
& I^{\alpha}\left[\frac{\partial^{\alpha} u(x, t)}{\partial t^{\alpha}}+a \frac{\partial^{\alpha-1} u(x, t)}{\partial t^{\alpha-1}}+b u(x, t)\right]=I^{\alpha}\left[c^{2} \frac{\partial^{2} u(x, t)}{\partial x^{2}}+h(x, t)\right], \\
& I^{\alpha}\left[\frac{\partial^{\alpha} u(x, t)}{\partial t^{\alpha}}\right]=I^{\alpha}\left[c^{2} \frac{\partial^{2} u(x, t)}{\partial x^{2}}-a \frac{\partial^{\alpha-1} u(x, t)}{\partial t^{\alpha-1}}-b u(x, t)+h(x, t)\right],
\end{aligned}
$$

by using (2.5), (2.6) and (2.7) we have

$$
u(x, t)=u(x, 0)+t u_{t}(x, 0)+I^{\alpha} h(x, t)+I^{\alpha}\left[c^{2} \frac{\partial^{2} u(x, t)}{\partial x^{2}}-a \frac{\partial^{\alpha-1} u(x, t)}{\partial t^{\alpha-1}}-b u(x, t)\right] .
$$

By using the initial conditions we have

$$
u(x, t)=f(x)+t g(x)+I^{\alpha} h(x, t)+I^{\alpha}\left[c^{2} \frac{\partial^{2} u(x, t)}{\partial x^{2}}-a \frac{\partial^{\alpha-1} u(x, t)}{\partial t^{\alpha-1}}-b u(x, t)\right] .
$$

Take

$$
\begin{aligned}
u_{0} & =f(x)+g(x) t+I^{\alpha} h(x, t) \\
u_{n+1} & =I^{\alpha}\left[c^{2} \frac{\partial^{2} u_{n}(x, t)}{\partial x^{2}}-a \frac{\partial^{\alpha-1} u_{n}(x, t)}{\partial t^{\alpha-1}}-b u_{n}(x, t)\right]
\end{aligned}
$$


Now, by iteration, we have

$$
\begin{aligned}
& u_{1}(x, t)=I^{\alpha}\left[c^{2} \frac{\partial^{2} u_{0}(x, t)}{\partial x^{2}}-a \frac{\partial^{\alpha-1} u_{0}(x, t)}{\partial t^{\alpha-1}}-b u_{0}(x, t)\right], \\
& u_{2}(x, t)=I^{\alpha}\left[c^{2} \frac{\partial^{2} u_{1}(x, t)}{\partial x^{2}}-a \frac{\partial^{\alpha-1} u_{1}(x, t)}{\partial t^{\alpha-1}}-b u_{1}(x, t)\right],
\end{aligned}
$$

by proceeding in this manner the solution of the nonhomogeneous time fractional telegraph equation is of the form

$$
u(x, t)=\sum_{n=0}^{\infty} u_{n}(x, t)=u_{0}(x, t)+u_{1}(x, t)+\cdots .
$$

\subsection{Numerical Examples.}

Example 3.1. Consider the equation (3.2) with $f(x)=0, g(x)=e^{x}, a=1, b=1, c=1$ and $0<x<1$ then the equation becomes

$$
\frac{\partial^{\beta} u(x, t)}{\partial t^{\beta}}+\frac{\partial^{\alpha} u(x, t)}{\partial t^{\alpha}}+u(x, t)=\frac{\partial^{2} u(x, t)}{\partial x^{2}}, t>0,1<\beta \leq 2, \frac{1}{2}<\alpha \leq 1 .
$$

with initial conditions

$$
u(x, 0)=0 \text { and } u_{t}(x, 0)=e^{x} .
$$

Then by Adomain decomposition method

$$
\begin{aligned}
u_{0}(x, t) & =e^{x} t \\
u_{1}(x, t) & =-e^{x} \frac{t^{\beta-\alpha+1}}{\Gamma(\beta-\alpha+2)}, \\
u_{2}(x, t) & =e^{x} \frac{t^{2 \beta-2 \alpha+1}}{\Gamma(2 \beta-2 \alpha+2)}, \\
u_{3}(x, t) & =-e^{x} \frac{t^{3 \beta-3 \alpha+1}}{\Gamma(3 \beta-3 \alpha+2)}, \text { etc }
\end{aligned}
$$

by proceeding in this manner the solution of the time fractional telegraph equation is of the form

$$
u(x, t)=\sum_{n=0}^{\infty} u_{n}(x, t)=u_{0}(x, t)+u_{1}(x, t)+\cdots
$$

Therefore,

$$
\begin{aligned}
u(x, t) & =e^{x} t-e^{x} \frac{t^{\beta-\alpha+1}}{\Gamma(\beta-\alpha+2)}+e^{x} \frac{t^{2 \beta-2 \alpha+1}}{\Gamma(2 \beta-2 \alpha+2)}-e^{x} \frac{t^{3 \beta-3 \alpha+1}}{\Gamma(3 \beta-3 \alpha+2)}+\ldots \\
& =t e^{x}\left[1-\frac{t^{\beta-\alpha}}{\Gamma(\beta-\alpha+2)}+\frac{t^{2 \beta-2 \alpha}}{\Gamma(2 \beta-2 \alpha+2)}-\frac{t^{3 \beta-3 \alpha}}{\Gamma(3 \beta-3 \alpha+2)}+\ldots\right] \\
& =t e^{x} E_{\beta-\alpha, 2}\left(-t^{\beta-\alpha}\right) .
\end{aligned}
$$




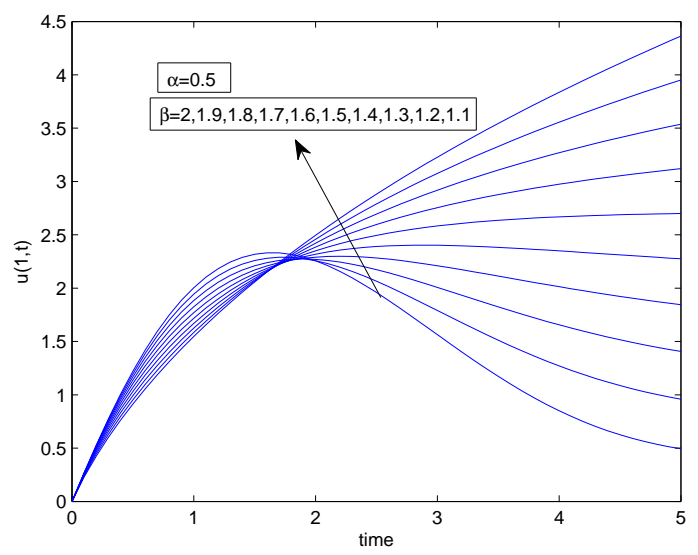

FIGURE 1. The graph is obtained for $\alpha=0.5$ and varying $\beta$.

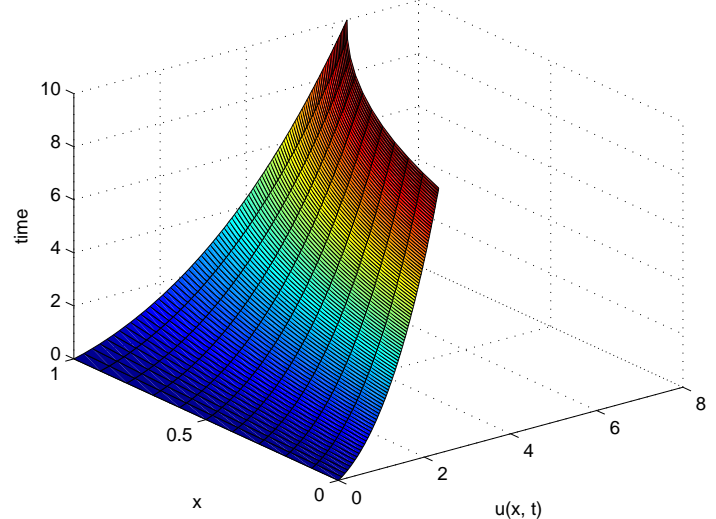

FIGURE 2. Surface plot

Therefore the solution of given telegraph equation is of the form $u(x, t)=t e^{x} E_{\beta-\alpha, 2}\left(-t^{\beta-\alpha}\right)$. The convergence $[1,26]$ of the series can be examined easily by simple ratio test. From Figure 1 it is observed that as time increases the solution $u(x, t)$ decays exponentially in integer order case compared to fractional order. Therefore the graph represents the decay of voltage or current in the transmission line. Surface plot of equation (3.5) is given in Figure 2. The exactness of the solution is clear by its convergence to the exact solution when $\alpha=1, \beta=2$ it coincides with the solution of classical telegraph equation.

$$
u(x, t)=\frac{e^{x}-e^{x-t}}{t} .
$$


Example 3.2. Consider the equation (3.4) with $a=1, b=1, f(x)=0, g(x)=0$ and $h(x, t)=\sinh x \frac{t^{n}}{\Gamma(n+1)}$ the equation becomes

$$
\frac{\partial^{\alpha} u(x, t)}{\partial t^{\alpha}}+\frac{\partial^{\alpha-1} u(x, t)}{\partial t^{\alpha-1}}+u(x, t)=\frac{\partial^{2} u(x, t)}{\partial x^{2}}+\sinh x \frac{t^{n}}{\Gamma(n+1)},
$$

with initial conditions

$$
u(x, 0)=0 \text { and } u_{t}(x, 0)=0 .
$$

Then by Adomain decomposition method we have

$$
\begin{aligned}
& u_{0}(x, t)=\sinh x \frac{t^{n+\alpha}}{\Gamma(n+\alpha+1)}, \\
& u_{1}(x, t)=-\sinh x \frac{t^{n+\alpha+1}}{\Gamma(n+\alpha+2)}, \\
& u_{2}(x, t)=\sinh x \frac{t^{n+\alpha+2}}{\Gamma(n+\alpha+3)}, \cdots .
\end{aligned}
$$

The solution in the series form is given by

$$
\begin{aligned}
u(x, t) & =\sinh x \frac{t^{n+\alpha}}{\Gamma(n+\alpha+1)}-\sinh x \frac{t^{n+\alpha+1}}{\Gamma(n+\alpha+2)}+\sinh x \frac{t^{n+\alpha+2}}{\Gamma(n+\alpha+3)}-\cdots, \\
& =\sinh x\left[\frac{t^{n+\alpha}}{\Gamma(n+\alpha+1)}-\frac{t^{n+\alpha+1}}{\Gamma(n+\alpha+2)}+\frac{t^{n+\alpha+2}}{\Gamma(n+\alpha+3)}-\cdots\right]
\end{aligned}
$$

The convergent $[1,26]$ of the series is examined by simple ratio test. From Figure 3 it is observed that as time increases the solution $u(x, t)$ decays exponentially in integer order case compared to fractional order. Therefore the graph represents the decay of voltage or current in the transmission line. Surface plot of equation (3.6) is given in Figure 4. The exactness of the solution is clear by its convergence to the exact solution when $\alpha=2$ it coincides with solution of classical telegraph equation

$$
u(x, t)=\sinh x\left[\frac{t^{n+2}}{\Gamma(n+3)}-\frac{t^{n+3}}{\Gamma(n+4)}+\frac{t^{n+4}}{\Gamma(n+5)}-\cdots\right] .
$$

\section{Decomposition Method by Laplace Transform}

Let us consider the time fractional telegraph equation (3.2) with nonhomogeneous term $h(x, t)$ and initial conditions (3.3). On taking Laplace transformation with respect to $t$ on both side of the equation (3.2) we have

$$
L_{t}\left(\frac{\partial^{\beta} u(x, t)}{\partial t^{\beta}}\right)+a L_{t}\left(\frac{\partial^{\alpha} u(x, t)}{\partial t^{\alpha}}\right)+b L_{t}(u(x, t))=L_{t}\left(c^{2} \frac{\partial^{2} u(x, t)}{\partial x^{2}}\right)+L_{t}(h(x, t))
$$




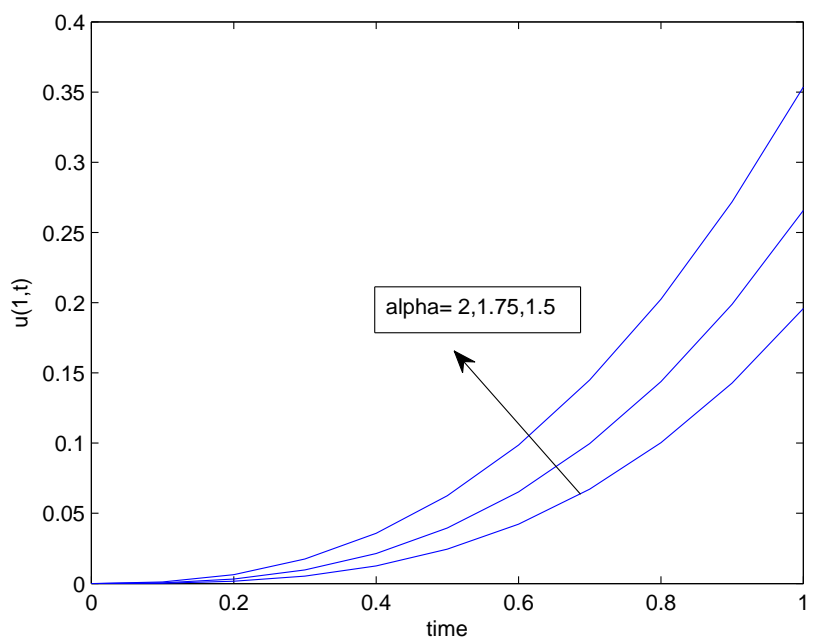

FIGURE 3. The graph is obtained for $\alpha=1.5,1.75,2$.

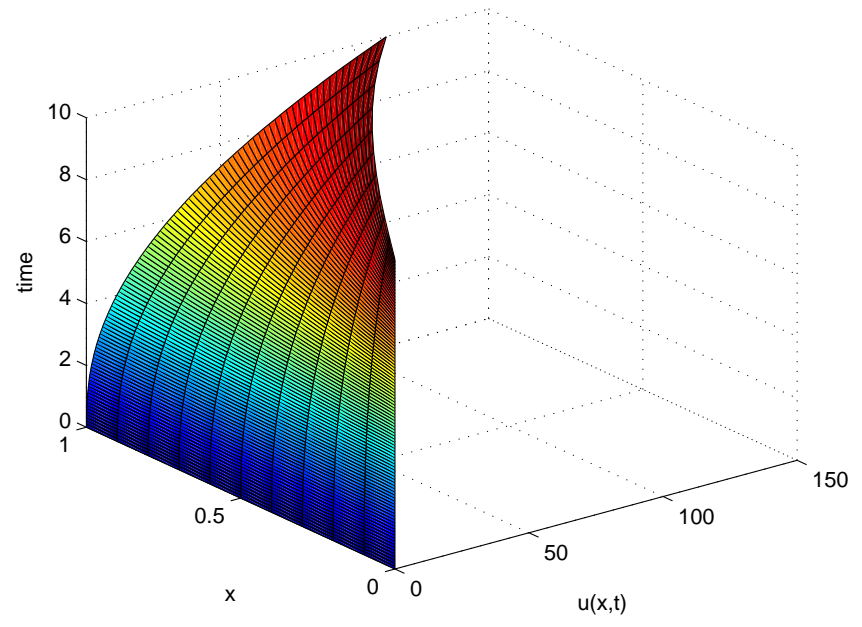

FIGURE 4. Surface plot

$$
\begin{aligned}
s^{\beta} L_{t}(u(x, t))-s^{\beta-1} u(x, 0)-s^{\beta-2} u_{t}(x, o) & +a L_{t}\left(\frac{\partial^{\alpha} u(x, t)}{\partial t^{\alpha}}\right)+b L_{t}(u(x, t)) \\
& =L_{t}\left(c^{2} \frac{\partial^{2} u(x, t)}{\partial x^{2}}\right)+L_{t}(h(x, t)),
\end{aligned}
$$


by using initial condition we get

$L_{t}(u(x, t))=\frac{f(x)}{s}+\frac{g(x)}{s^{2}}+\frac{1}{s^{\beta}} L_{t}(h(x, t))+\frac{1}{s^{\beta}} L_{t}\left(c^{2} \frac{\partial^{2} u(x, t)}{\partial x^{2}}-a \frac{\partial^{\alpha} u(x, t)}{\partial t^{\alpha}}-b u(x, t)\right)$,

then taking inverse Laplace transformation

$u(x, t)=L_{t}^{-1}\left[\frac{f(x)}{s}+\frac{g(x)}{s^{2}}+\frac{1}{s^{\beta}} L_{t}(h(x, t))\right]+L_{t}^{-1} \frac{1}{s^{\beta}}\left[L_{t}\left(c^{2} \frac{\partial^{2} u(x, t)}{\partial x^{2}}-a \frac{\partial^{\alpha} u(x, t)}{\partial t^{\alpha}}-b u(x, t)\right)\right]$,

on applying the Adomain decomposition method we have,

$$
\begin{aligned}
u_{0}(x, t) & =L_{t}^{-1}\left[\frac{f(x)}{s}+\frac{g(x)}{s^{2}}+\frac{1}{s^{\beta}} L_{t}(h(x, t))\right] \\
u_{n+1}(x, t) & =L_{t}^{-1} \frac{1}{s^{\beta}}\left[L_{t}\left(c^{2} \frac{\partial^{2} u(x, t)}{\partial x^{2}}-a \frac{\partial^{\alpha} u(x, t)}{\partial t^{\alpha}}-b u(x, t)\right)\right] .
\end{aligned}
$$

On calculating $u_{1}(x, t), u_{2}(x, t), \cdots$ we get the series solution of the form

$$
u(x, t)=\sum_{n=0}^{\infty} u_{n}(x, t)=u_{0}(x, t)+u_{1}(x, t)+\cdots
$$

Example 4.1. Considering the equation (3.2) with $f(x)=0, g(x)=e^{x}, a=1, b=1, c=1$ and $0<x<1$ the equation becomes

$$
\frac{\partial^{\beta} u(x, t)}{\partial t^{\beta}}+\frac{\partial^{\alpha} u(x, t)}{\partial t^{\alpha}}+u(x, t)=\frac{\partial^{2} u(x, t)}{\partial x^{2}}, t>0,1<\beta \leq 2, \frac{1}{2}<\alpha \leq 1 .
$$

with initial conditions

$$
u(x, 0)=0 \text { and } u_{t}(x, 0)=e^{x} .
$$

By taking Laplace transform on both sides we get,

$$
\begin{array}{r}
s^{\beta} L_{t}(u(x, t))-s^{\beta-1} u(x, 0)-s^{\beta-2} u_{t}(x, o)+L_{t}\left(\frac{\partial^{\alpha} u(x, t)}{\partial t^{\alpha}}\right)+L_{t}(u(x, t)) \\
=L_{t}\left(\frac{\partial^{2} u(x, t)}{\partial x^{2}}\right),
\end{array}
$$

on applying the conditions we have

$$
L_{t}(u(x, t))=\frac{e^{x}}{s^{2}}+L_{t} \frac{1}{s^{\beta}}\left(\frac{\partial^{2} u(x, t)}{\partial x^{2}}-\frac{\partial^{\alpha} u(x, t)}{\partial t^{\alpha}}-u(x, t)\right),
$$

then taking inverse Laplace transform we get,

$$
u(x, t)=L_{t}^{-1}\left[\frac{e^{x}}{s^{2}}\right]+L_{t}^{-1}\left[L_{t}\left(\frac{\partial^{2} u(x, t)}{\partial x^{2}}-\frac{\partial^{\alpha} u(x, t)}{\partial t^{\alpha}}-u(x, t)\right)\right] .
$$


Therefore, by Adomain decompositioin method we have

$$
\begin{aligned}
& u_{0}(x, t)=t e^{x} \\
& u_{1}(x, t)=-e^{x} \frac{t^{\beta-\alpha+1}}{\Gamma(\beta-\alpha+2)},
\end{aligned}
$$

by proceeding in this manner the solution of the time fractional telegraph equation is of the form $u(x, t)=t e^{x} E_{\beta-\alpha, 2}\left(-t^{\beta-\alpha}\right)$.

Example 4.2. If $a=1, b=1, f(x)=0, g(x)=0$ and $h(x, t)=\sinh x \frac{t^{n}}{\Gamma(n+1)}$ in (3.4) and (3.3) then by taking Laplace transform on both side we have

$$
L_{t}(u(x, t))=\frac{1}{s^{\alpha}} L_{t}\left(\sinh x \frac{t^{n}}{\Gamma(n+1)}\right)+\frac{1}{s^{\alpha}} L_{t}\left(\frac{\partial^{2} u(x, t)}{\partial x^{2}}-\frac{\partial^{\alpha-1} u(x, t)}{\partial t^{\alpha-1}}-u(x, t)\right) .
$$

By taking inverse Laplace transform and on calculating $u_{0}(x, t), u_{1}(x, t), \cdots$ by Adomain decomposition method we get

$$
\begin{aligned}
& u_{0}(x, t)=\sinh x \frac{t^{n+\alpha}}{\Gamma(n+\alpha+1)}, \\
& u_{1}(x, t)=-\sinh x \frac{t^{n+\alpha+1}}{\Gamma(n+\alpha+2)},
\end{aligned}
$$

by proceeding in this manner the solution of the equation is of the form

$$
u(x, t)=\sinh x\left[\frac{t^{n+\alpha}}{\Gamma(n+\alpha+1)}-\frac{t^{n+\alpha+1}}{\Gamma(n+\alpha+2)}+\frac{t^{n+\alpha+2}}{\Gamma(n+\alpha+3)}-\ldots\right]
$$

Example 4.3. (Fourier and Laplace Transformation Method) Huang[16] effectively applied Fourier and Laplace transformation method to time fractional telegraph equation. Here, a numerical example is given to illustrate this method. Consider the equation (3.2) by taking, $a=1, b=1, c=1$ the equation becomes

$$
\frac{\partial^{2 \alpha} u(x, t)}{\partial t^{2 \alpha}}+\frac{\partial^{\alpha} u(x, t)}{\partial t^{\alpha}}+u(x, t)=\frac{\partial^{2} u(x, t)}{\partial x^{2}}, t>0,0<x \leq L
$$

with initial conditions

$$
u(x, 0)=0 \text { and } u_{t}(x, 0)=e^{x} .
$$

by taking Fourier and Laplace transform with respect to the variables $x$ and $t$ we get the solution of (4.1) of the form

$$
u(x, t)=\frac{2}{L} \sum_{n=1}^{\infty} \sin \left(\frac{n \pi x}{L}\right)\left[c_{1} E_{\alpha, 2}\left(\lambda_{+} t^{\alpha}\right)-c_{2} E_{\alpha, 2}\left(\lambda_{-} t^{\alpha}\right)\right] \int_{0}^{L} e^{x} \sin \left(\frac{n \pi x}{L}\right) \mathrm{d} x .
$$


where, $\lambda_{+}=\frac{-1+\sqrt{1-4\left(1+\frac{n^{2} \pi^{2}}{4}\right)}}{2}, \lambda_{-}=\frac{-1-\sqrt{1-4\left(1+\frac{n^{2} \pi^{2}}{4}\right)}}{2}, c_{1}=\frac{\lambda_{+}}{\lambda_{+}-\lambda_{-}}$and $c_{2}=\frac{\lambda_{-}}{\lambda_{+}-\lambda_{-}}$

\section{CONCLUSiON}

The solution obtained from decomposition method by Laplace transform coincides with the solution obtained from only decomposition method. Also it is necessary to note here that by knowing initial condition alone we can get the exact solution and so it is more convenient than double Laplace transform method where both initial and boundary conditions are needed. Therefore Adomain decomposition method is an easier way to obtain exact solution for many fractional partial differential equations. The solution we obtained here is a closed form series solution in terms of Mittag-Leffler function. The series solution of time fractional telegraph equation is analysed by plotting the solution for various fractional order and convergence is verified.

\section{ACKNOWLEDGMENTS}

The author's thank the reviwers for their valuable suggestion.

\section{REFERENCES}

[1] K. Abboui and Y. Cherruaut, New Ideas for Proving Convergence of Decomposition Methods, Computers and Mathematics with Application, 29(1996), 103-108.

[2] G. Adomain, Solving frontier problems of Physics, Kluwar, Dordrecht, 1994.

[3] G. Adomain, A Rieview of the Decomposition Method in Applied Mathematics, Journal of Mathematical Analysis and Application, 135(1988), 501-544.

[4] O.P. Agrawal, Solution for a Fractional Diffusion-Wave Equation Defined in Dounded Domain, Journal of Nonlinear Dynamics, 29(2002), 145-155.

[5] A.M.O. Anwar, F. Jarad, D. Baleanu and F. Ayaz, Fractional Caputo Heat Equation within the Double Laplace Transform, Romanian Journal of Physics, 58(2013), 15-22.

[6] J. Biazar, E. Babolian and R. Islam, Solution of the System of Volterra Integral Equations of the First Kind by Adomian Decomposition Method, Applied Mathematics and Computation,139(2003), 249-258.

[7] J. Biazar, E. Babolian and R. Islam, Solution of the System of Ordinary Differential Equations by Adomian Decomposition Method, Applied Mathematics and Computation, 147(2004), 713-719.

[8] J. Biazar and H. Ebrahimi, An Approximation to the Solution of Telegraph Equation by Adomain Decomposition Method, International Mathematics Fourm, 2(2007), 2231-2236.

[9] R.C. Cascaval, E.C. Eckstein, C.L. Frota and J.A. Goldstein,Fractional Telegraph Equations, Journal of Mathematical Analysis and Applications, 276(2002), 145-159.

[10] V. Daftardar-Grjji and H. Jafari, Boundary Value Problems for Fractional Diffusion-Wave Equation, Australian Journal of Mathematical Analysis and Applications, 3(2006), 1449-5910.

[11] V. Daftardar-Gejji and H. Jafari, Adomain Decomposition Method: A Tool for Solving Fractional Differential Equations, Journal of Mathematical Analysis and Applications, 301(2005), 508-518.

[12] V. Daftardar-Grjji and H. Jafari, Solving a System of Nonlinear Fractional Differential Eqautions using Adomain Decomposition Method, Journal of Computational and Applied Mathematics, 196(2006), 644-651. 
[13] V. Daftardar-Gejji, A. Babakhani and R. Islam, Solution of the System of Ordinary Differential Equations by Adomain Decomposition Method, Applied Mathematics and Computation, 147(2004), 713-719.

[14] E.C. Eckstein, J.A. Goldstein and M. Leggas, The Mathematics of Suspensions, Kac Walks and Asymptotic Analyticity, Electronic Journal of Differential Equations, 03(1999), 39-50.

[15] H. Eltayeb and A. Kilicman, A Note on Double Laplace transformation and Telegraphic Equations, Abstract and applied analysis, 2013(2013), 1-6.

[16] F. Huang, Analytical Solution for the Time Fractional Telegraph Equation by Laplace and Fourier Transform, Journal of Applied Mathematics, 2009(2009), 1-9.

[17] M. Javidi and B. Ahmed, Numerical Solution of Fractional Partial Differential Equation by Numerical Laplace Inverse Technique, Advances in Difference Equations,375(2013), 1-18.

[18] K.S. Miller and B. Ross, An introduction to the fractional calculus and fractional differential equations, Wiley and Sons, New York, 1993.

[19] D. Kaya, A New Approach to the Telegraph Equation: An Application of the Decomposition Method, Bulletin of the Institute of Mathematics Academics Sinica, 28(2000), 51-57.

[20] Y. Khan, J. Diblik, N. Faraz and Z. Smarda, A Efficient New Perturbative Laplace Method for Space-Time Fractional Telegraph Equation, Advances in Difference Equations, 204(2012), 1-9.

[21] A. Kilbas, H.M. Srivastava and J.J. Trujillo, Theory and application of fractional differential equations, Elsevier, Amstrdam, 2006.

[22] S. Momani, Analytic and Approximate Solutions of the Space and Time Fractional Telegraph Equations, Applied Mathmatics and Computation, 170(2005), 1126-1134.

[23] K.B. Oldham and J. Spanier, The fractional calculus: Theory and application of differentiation and integration to arbitrary order, Academic Press, New York, 1974.

[24] E. Orsingher and L. Beghin, Time Fractional Telegraph Equations and Telegraph Processes with Brownian Time, Probability Theory and Related Fields, 128(2004), 141-160.

[25] E. Orsingher and X. Zhao, The Space Fractional Telegraph Equation and the Related Fractional Telegraph Process, Chinese Annals of Mathematics Series B, 24(2003), 45-56.

[26] J. Paneva-Konovska, Series in Mittag-Leffler Functions: Inequalities and Convergent Theorems, Fractional Calculus and Applied Analysis, 13(2010), 403-414.

[27] V. Parthiban and K. Balachandran, Solutions of System of Fractional Partial Differential Equation, Application and Applied Mathematics: An International Journal, 8 (2013), 289-304.

[28] I. Podlubny, Fractional differential equations, Academic Press, New York, 1999.

[29] U. Hayat and S.T. Mohyud-Din, Homotopy Perturbation Technique for Time Fractional Telegraph Equations, International Journal of Modern Theoretical Physics, 2(2013), 33-41.

[30] A. Sevimlican, An Approximation to Solution of Space and Time Fractional Telegraph Equations by Variational Iteration Method, Mathematical Problems in Engineering, 2010(2010), 1-10.

[31] A.M. Wazwaz, A Reliable Technique for Solving the Wave Equation in Infinite One-dimensional Medium, Applied Mathematics and Computation, 92(1998), 1-7.

[32] A. Yidirim, Homotopy Perturbation Method for Solving the Space and Time Fractional Telegraph Equations, International Journal of Computer Mathematics, 87(2010), 2998-3006.

[33] Z. Odibat and S. Momani, Numerical Methods for Nonlinear Partial Differential Equations of Fractional Order, Applied Mathematical Modeling, 32(2008), 28-29. 